\title{
A Comparison Study on Peridynamic Models Using Irregular Non- uniform Spatial Discretization
}

\author{
Hailong Chen
}

March 2019

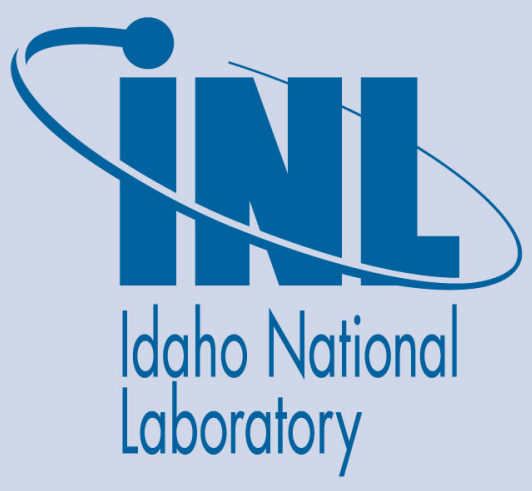

The INL is a U.S. Department of Energy National Laboratory operated by Battelle Energy Alliance 


\title{
A Comparison Study on Peridynamic Models Using Irregular Non-uniform Spatial Discretization
}

\author{
Hailong Chen
}

March 2019

Idaho National Laboratory Idaho Falls, Idaho 83415

http://www.inl.gov

Prepared for the U.S. Department of Energy Office of Nuclear Energy Under DOE Idaho Operations Office

Contract DE-AC07-05ID14517 


\title{
A Comparison Study on Peridynamic Models Using Irregular Non-uniform Spatial Discretization
}

\author{
Hailong Chen ${ }^{1}$
}

Fuels Modeling and Simulation Department, Idaho National Laboratory, Idaho Falls, ID 83402

\begin{abstract}
The applicability of peridynamic models to problems with irregularly non-uniformly discretized solution domain is critical. In this study, a systematic comparison study on results predicted using eight different peridynamic models, including bond-based, ordinary state-based and nonordinary state-based mechanics and heat conduction models, for three different types of mechanical problems, including thermal, mechanics and coupled thermo-mechanics, with irregular non-uniform spatial discretization are performed. It's found that for the case of irregular but semi-uniform spatial discretization, all these models yield good predictions compared to analytical local solutions. For the case of irregular and non-uniform spatial discretization, models formulated specifically for this configuration give much better results than the conventional formulations which don't consider the neighborhood difference among material points in the spatial discretization. For either cases of spatial discretization, the bond-associated correspondence material model predicts the most accurate results.

Keywords: Peridynamics; Bond-based models; State-based models; Correspondence models; Irregular spatial discretization
\end{abstract}

\section{Introduction}

Peridynamic models have clear advantages in materials failure modeling due to their intrinsic integral formulation of the conventional stress divergence kernel in classical continuum

\footnotetext{
${ }^{1}$ Tel.: +12085262542.

E-mail address: hailong.chen@inl.gov.
} 
mechanics. With the formulation of integro-differential equation of motion, stress singularity related issues for problems involve spatial discontinuities such as cracks are better avoided in peridynamics theory. Besides, for modeling of any fracture problems, crack initiation can be handled easily and effectively using interaction removal between initially interacting material points in a discretized spatial domain. Crack nucleation, propagation, branching and coalescence are the natural outcomes of this interaction removal process. Except a one-dimensional interaction removal criterion, such as critical stretch criterion, no externally devised complex failure criteria are required, which is not the case for other continuum based numerical methods, such as Finite Element Methods (FEMs) and eXtended FEM (XFEM). For extensive literature survey on peridynamics theory and it application, one can refer to works by Bobaru et al. [1], and Madenci and Oterkus [2].

With a few exceptions, most previous peridynamic applications employ regular uniform discretization of the solution domain. Although regular uniform discretization is easy to generate, it can result in excessive computational expense because the refinement level is driven by the maximum refinement needed anywhere in the solution domain. On one hand, local refinement is an obvious solution to this issue - concentrating material points at the locations where they are most needed for solution accuracy. On the other hand, local refinement may bring in the issue of unbalanced interaction, i.e., ghost force effect [3].

Computational cost is not the only drawback to regular uniform discretization, however, as they may influence crack paths due to dependencies on the orientation of the grid, as reported by Chen et al. [4] and Dipasquale et al. [5]. Moreover, regular discretization may influence damage initiation sites because of grid symmetry [6] and inaccurate geometry representation [7], [8] in the computational model. These phenomena are particularly evident when considering domains 
with complex or curved geometries. To overcome these aforementioned shortcomings, capability of applying peridynamic models to irregular and non-uniform spatial discretization with variable horizon size becomes critical.

So far, there was no study on investigation of the performance of different peridynamic models when irregular non-uniform spatial discretization is used. In this paper, a systematic comparison on results predicted by eight different bond-based and state-based peridynamic models for irregular non-uniform spatial discretization is performed. Modeling failure of solids is out of the scope of current study, since valid peridynamic failure criteria for irregular spatial discretization is still under development and application of regular uniform grid based failure criteria to problems with irregular non-uniform spatial discretization is arguable. Also, since the purpose of this study is to quantitatively investigate the effects of irregularity and non-uniformity of the spatial discretization on prediction accuracy of peridynamic models, some mechanics and heat conduction problems with analytical solutions are utilized. There is no doubt that these physical problems can be handled easily and efficiently using other numerical methods, such as FEM. The remainder of this paper is organized as follows: Section 2 briefly reviews peridynamic models that will be used in this study. These models include bond-based models using conventional constant parameters [9], [10] and variable parameters [8], ordinary state-based models using conventional constant parameters [2] and variable parameters [11], conventional correspondence model stabilized with spring-like force [12], and self-stabilized bond-associated correspondence model [13]. Section 3 presents the prediction and comparison among these models using three different types of problems. Discussion and conclusion are presented in Section 4 .

\section{Peridynamic models: a brief review}


In this section, details about different peridynamic mechanics and heat conduction models are reviewed. It should be noted that all these peridynamic models are available in the open-source MOOSE package [14] developed at Idaho National Laboratory.

\subsection{Mechanics models}

In peridynamic theory, the equation of motion for a material point $\mathbf{X}$ in the reference configuration at time ${ }^{t}$ is given by

$$
\rho(\mathbf{X}) \ddot{\mathbf{u}}(\mathbf{X}, t)=\int_{H_{\mathbf{X}}} \mathbf{f}\left(\mathbf{X}, \mathbf{X}^{\prime}, t\right) d V_{\mathbf{X}^{\prime}}+\mathbf{b}(\mathbf{X}, t) \quad \forall(\mathbf{X}, t) \in \Omega \times(0, \tau)
$$

MERGEFORMAT ()

where $\rho(\mathbf{X})$ is mass density, $\mathbf{u}(\mathbf{X}, t)$ is the displacement vector, $\mathbf{f}\left(\mathbf{X}, \mathbf{X}^{\prime}, t\right)$ is the density per unit volume of the pairwise force vector for bond connecting material points $\mathbf{X}$ and $\mathbf{X}^{\prime}$, and $\mathbf{b}(\mathbf{X}, t)$ is external force density vector.

Depend on the formulation, i.e., bond-based or state-based, the calculation of force density vector will be different. For bond-based formulation, the force density vector of a bond depends only on its own deformation state. While for state-based formulation, besides its own deformation state, all deformation states connecting these two material points with their family members collectively contribute to the calculation of the bond force density vector.

\subsubsection{Bond-based models}

For bond-based peridynamic mechanics models, the force density vector $\mathbf{f}\left(\mathbf{X}, \mathbf{X}^{\prime}, t\right)$ can be written as

$$
\mathbf{f}\left(\mathbf{X}, \mathbf{X}^{\prime}, t\right)=c\left(\mathbf{X}, \mathbf{X}^{\prime}\right) s\left(\mathbf{X}, \mathbf{X}^{\prime}, t\right) \mathbf{M}
$$

MERGEFORMAT ()

where the unit vector in the direction of deformed bond from $\mathbf{X}$ to $\mathbf{X}^{\prime}$ is 


$$
\mathbf{M}=\frac{\mathbf{x}^{\prime}\left(\mathbf{X}^{\prime}, t\right)-\mathbf{x}(\mathbf{X}, t)}{\left|\mathbf{x}^{\prime}\left(\mathbf{X}^{\prime}, t\right)-\mathbf{x}(\mathbf{X}, t)\right|}
$$

MERGEFORMAT ()

and the bond stretch is

$$
s\left(\mathbf{X}, \mathbf{X}^{\prime}, t\right)=\frac{\left|\mathbf{x}^{\prime}\left(\mathbf{X}^{\prime}, t\right)-\mathbf{x}(\mathbf{X}, t)\right|-\left|\mathbf{X}^{\prime}-\mathbf{X}\right|}{\left|\mathbf{X}^{\prime}-\mathbf{X}\right|}
$$

MERGEFORMAT ()

The constant bond micro-modulus based on regular uniform spatial discretization is [7], [9]

$$
c\left(\mathbf{X}, \mathbf{X}^{\prime}\right)=\frac{6 D K}{\pi \delta^{D+1}}
$$

MERGEFORMAT ()

with $K$ is the material bulk modulus, $D$ is the dimensionality number with $D=2$ for two dimensional analysis and $D=3$ for three-dimensional analysis, and $\delta$ is the horizon radius. The variable bond micro-modulus based on irregular spatial discretization is [8]

$$
c\left(\mathbf{X}, \mathbf{X}^{\prime}\right)=\frac{D^{2} K}{\left|\mathbf{X}^{\prime}-\mathbf{X}\right|}\left(\frac{\sum_{m=1}^{N} V\left(\mathbf{X}_{m}\right)+\sum_{n=1}^{N^{\prime}} V\left(\mathbf{X}_{n}\right)}{\sum_{m=1}^{N} V\left(\mathbf{X}_{m}\right) \sum_{n=1}^{N^{\prime}} V\left(\mathbf{X}_{n}\right)}\right)
$$

MERGEFORMAT ()

where $V\left(\mathbf{X}_{m}\right)$ is volume of material point $\mathbf{X}_{m}$ who is a family member of material point $\mathbf{X}$, and $N$ is the total number of neighboring material points within material point $\mathbf{X}$ 's family. The same definition applies to material point $\mathbf{X}^{\prime}$.

It should be noted that Eq. can be recovered from Eq. when regular uniform spatial discretization is used [8]. 


\subsubsection{Ordinary state-based models}

For ordinary state-based peridynamic mechanics models, the force density vector can be generally expressed as

$$
\mathbf{f}\left(\mathbf{X}, \mathbf{X}^{\prime}, t\right)=\underline{\mathbf{t}}[\mathbf{X}, t]\left\langle\mathbf{X}^{\prime}-\mathbf{X}\right\rangle-\underline{\mathbf{t}}\left[\mathbf{X}^{\prime}, t\right]\left\langle\mathbf{X}-\mathbf{X}^{\prime}\right\rangle
$$

MERGEFORMAT ()

where $\underline{\mathbf{t}}[\mathbf{X}, t]$ is the force vector state that material point $\mathbf{X}^{\prime}$ exerts on material point $\mathbf{X}$, and $\underline{\mathbf{t}}\left[\mathbf{X}^{\prime}, t\right]$ is the force vector state that material point $\mathbf{X}$ exerts on material point $\mathbf{X}^{\prime}$.

There are two different formulations for ordinary state-based peridynamic model in the literature. A detailed comparison of these two formulations for elasticity problem can be found at Ref. [15].

For this study, we adopt the formulations proposed by Madenci and Oterkus [2] and Hu et al. [11].

According to Madenci and Oterkus [2], the force vector state can be written as

$$
\underline{\mathbf{t}}=2 \delta\left(d \mathbf{M} \cdot \frac{\xi}{|\xi|} a \theta+b s\right) \mathbf{M}
$$

MERGEFORMAT ()

where $a, b, d$ are peridynamic constants which can be derived in terms of material constants and horizon radius $\delta, \xi=\mathbf{X}^{\prime}-\mathbf{X}$ is the relative position vector state, and $\theta$ is the dilatation at a material point and is defined as

$$
\theta=d \delta \int_{H_{\mathbf{X}}} s\left(\mathbf{X}, \mathbf{X}^{\prime}, t\right) \frac{\xi}{|\xi|} \cdot \mathbf{M} d V
$$

MERGEFORMAT ()

The peridynamic constant $a$ is spatial discretization independent and can be expressed as 


$$
a=\frac{(2 D-3) K-(3 D-4) \mu}{2}
$$

MERGEFORMAT ()

where ${ }^{\mu}$ is the shear modulus, $K$ is the bulk modulus and $D$ is the dimensionality number. For regular uniform spatial discretization [2],

$$
\begin{aligned}
& b=\frac{(9 D-12) \mu}{(D-1) \pi \delta^{D+2}} \\
& d=\frac{7 D-12}{(3 D-5) \pi \delta^{D+1}}
\end{aligned}
$$

and for irregular non-uniform spatial discretization [11],

$$
\begin{aligned}
& \delta_{\mathbf{X}} b_{\mathbf{X}}+\delta_{\mathbf{X}^{\prime}} b_{\mathbf{X}^{\prime}}=\frac{D(K-2 a)}{2|\xi|}\left(\delta_{\mathbf{x}} d_{\mathbf{X}}+\delta_{\mathbf{X}^{\prime}} d_{\mathbf{X}^{\prime}}\right) \\
& d=\frac{D}{\delta \int_{H} 1 d V}
\end{aligned}
$$

As have been shown in Ref. [11], the peridynamic constants shown in Eqs. and for irregular non-uniform spatial discretization reduce to Eqs. and when regular uniform spatial discretization is used.

\subsubsection{Non-ordinary state-based models}

For peridynamic non-ordinary state-based models, or correspondence material models, the force 
density vector has following generally expression

$$
\mathbf{f}\left(\mathbf{X}, \mathbf{X}^{\prime}, t\right)=\underline{\mathbf{T}}[\mathbf{X}, t]\left\langle\mathbf{X}^{\prime}-\mathbf{X}\right\rangle-\underline{\mathbf{T}}\left[\mathbf{X}^{\prime}, t\right]\left\langle\mathbf{X}-\mathbf{X}^{\prime}\right\rangle
$$

MERGEFORMAT ()

where $\underline{\mathbf{T}}[\mathbf{X}, t]$ is the force vector state that material point $\mathbf{X}^{\prime}$ exerts on material point $\mathbf{X}$, and $\underline{\mathbf{T}}\left[\mathbf{X}^{\prime}, t\right]$ is the force vector state that material point $\mathbf{X}$ exerts on material point $\mathbf{X}^{\prime}$.

The conventional peridynamic correspondence material model has the issue of material instability [16]. Stabilization scheme is required in order for the conventional correspondence material model to yield meaningful results. In conventional correspondence material model, the force state with penalty force discussed in Ref. [12] can be written as

$$
\underline{\mathbf{T}}[\mathbf{X}, t]\left\langle\mathbf{X}^{\prime}-\mathbf{X}\right\rangle=\omega\langle\xi\rangle \mathbf{P} \cdot(\mathbf{K})^{-1} \cdot \boldsymbol{\xi}+C_{Z E C} E \Delta x \omega\langle\xi\rangle\left(\mathbf{u}\left(\mathbf{X}^{\prime}, t\right)-\mathbf{u}(\mathbf{X}, t)\right)
$$

MERGEFORMAT ()

where $C_{Z E C}$ is zero-energy control parameter, $E$ is material Young's modulus, $\Delta x$ is material point spacing.

Rather than using externally devised stabilization force, one effective way to inherently remove this material instability is to formulate a bond-associated deformation gradient [13]. In this selfstabilized bond-associated correspondence material model, the force state of bond connecting material point $\mathbf{X}$ and $\mathbf{X}^{\prime}$ is formulated based on the bond-associated horizon and is given as

$$
\underline{\mathbf{T}}^{b}[\mathbf{X}, t]\left\langle\mathbf{X}^{\prime}-\mathbf{X}\right\rangle=\frac{\int_{H_{\mathbf{X}} \cap h_{\mathbf{X}^{\prime}}} 1 d V_{\mathbf{X}^{\prime}}}{\int_{H_{\mathbf{X}}} 1 d V_{\mathbf{X}^{\prime}}} \omega\langle\xi\rangle \mathbf{P}^{b} \cdot\left(\mathbf{K}^{b}\right)^{-1} \cdot \boldsymbol{\xi}
$$

MERGEFORMAT ()

where $h_{\mathbf{X}^{\prime}}$ is the bond-associated horizon for bond $\xi$ at material point $\mathbf{X}^{\prime}, \mathbf{P}^{b}$ is the bondassociated first Piola-Kirchhoff stress at material point $\mathbf{X}$, and $\mathbf{K}^{b}$ is the bond-associated shape 
tensor at material point $\mathbf{X}$.

\subsection{Heat conduction models}

Similar to the peridynamic mechanics equation of motion, the peridynamic heat conduction equation at material point $\mathbf{X}$ at time ${ }^{t}$ is formulated in its integral form as [10]

$$
\rho(\mathbf{X}) C \dot{T}(\mathbf{X}, t)=\int_{H_{\mathbf{X}}} f_{h}\left(\mathbf{X}, \mathbf{X}^{\prime}, t\right) d V_{\mathbf{X}^{\prime}}+q(\mathbf{X}, t) \quad \forall(\mathbf{X}, t) \in \Omega_{r} \times(0, \tau)
$$

MERGEFORMAT ()

where $C$ is the heat capacity, $T(\mathbf{X}, t)$ is the temperature, $f_{h}\left(\mathbf{X}, \mathbf{X}^{\prime}, t\right)$ is the thermal response function, and $q(\mathbf{X}, t)$ is the heat source or sink.

The thermal response function can be expressed in terms of the temperature at the two material points as

$$
f_{h}\left(\mathbf{X}, \mathbf{X}^{\prime}, t\right)=k\left(\mathbf{X}, \mathbf{X}^{\prime}\right) \frac{T\left(\mathbf{X}^{\prime}, t\right)-T(\mathbf{X}, t)}{\left|\mathbf{X}^{\prime}-\mathbf{X}\right|}
$$

MERGEFORMAT ()

with $k$ is the peridynamic bond micro-conductivity.

The constant bond micro-conductivity based on regular uniform spatial discretization is [10]

$$
k\left(\mathbf{X}, \mathbf{X}^{\prime}\right)=\frac{6 \lambda}{\pi \delta^{D+1}}
$$

MERGEFORMAT ()

with $\lambda$ is the material thermal conductivity, $D$ is the dimensionality number.

The variable bond micro-conductivity based on irregular discretization is [8]

$$
k\left(\mathbf{X}, \mathbf{X}^{\prime}\right)=\frac{D \lambda}{\left|\mathbf{X}^{\prime}-\mathbf{X}\right|}\left(\frac{\sum_{m=1}^{N} V\left(\mathbf{X}_{m}\right)+\sum_{n=1}^{N^{\prime}} V\left(\mathbf{X}_{n}\right)}{\sum_{m=1}^{N} V\left(\mathbf{X}_{m}\right) \sum_{n=1}^{N^{\prime}} V\left(\mathbf{X}_{n}\right)}\right)
$$


Again, Eq. can be recovered from Eq. when regular uniform spatial discretization is used.

2.3. Coupling between peridynamic heat conduction and mechanics models

In this study, the coupling between heat conduction model and mechanics models is introduced via the thermal effect on the total stretch, dilatation and strain calculation.

For bond-based mechanics models, the bond elastic stretch considering the thermal effect can be calculated as

$$
s_{\text {elastic }}\left(\mathbf{X}, \mathbf{X}^{\prime}, t\right)=s\left(\mathbf{X}, \mathbf{X}^{\prime}, t\right)-\alpha \frac{\Delta T\left(\mathbf{X}^{\prime}, t\right)+\Delta T(\mathbf{X}, t)}{2}
$$

MERGEFORMAT ()

where $\alpha$ is the thermal expansion coefficient and $\Delta T$ is the temperature difference with respect to the stress free temperature at a material point.

For ordinary state-based peridynamic mechanics model, besides the coupling of thermal effect into the bond stretch, the dilatation at material point also needs to consider the thermal effect. The expression for the dilatation shown in Eq. can be updated as

$$
\theta=d \delta \int_{H_{\mathbf{X}}} S_{\text {elastic }} \frac{\xi}{|\xi|} \cdot \mathbf{M} d V+3 \alpha \Delta T
$$

MERGEFORMAT ()

The force vector state of ordinary state-based model for a coupled thermo-mechanical analysis becomes

$$
\underline{\mathbf{t}}=2 \delta\left(d \mathbf{M} \cdot \frac{\xi}{|\xi|}\left(a \theta-\frac{1}{2} a_{2} \Delta T\right)+b s\right) \mathbf{M}
$$


where $a_{2}=2 D \alpha a$ and $a$ is defined in Eq. .

For non-ordinary state-based models, the elastic strain tensor at a material point considering the thermal effect can be calculated as

$$
\mathbf{E}=\frac{1}{2}\left(\mathbf{F}^{T} \mathbf{F}-\mathbf{I}\right)-\alpha \Delta T \mathbf{I}
$$

MERGEFORMAT ()

where $\mathbf{F}$ is the deformation gradient and $\mathbf{I}$ is the identity matrix.

For fracture models, the bond breakage status determined by bond stretch in mechanics models affect the thermal response between material points in the heat conduction models. Since the focus of this study is to investigate the effect of spatial discretization on peridynamic models in terms of temperature and displacements prediction accuracy, the one-way coupling scheme, i.e., thermal affecting mechanics, is used throughout all the case studies.

\section{Case studies}

In order to better assess the performance of peridynamic models outlined in Section 2 for irregular spatial discretization, two types of geometry with different irregularity and nonuniformity are employed in this section, as shown in Figure 1. The square plate has length of 0.1 $\mathrm{m}$, while the circular plate has radius of $0.0041 \mathrm{~m}$. For the square plate, two local refinements are used, with one being regular and the other being irregular, which result in a total of 20,197 material points. The rectangular region with regular refinement has the dimension of $0.03 \mathrm{~m}$ by $0.04 \mathrm{~m}$, with center at $(-0.025,0)$. The elliptical region with irregular refinement has the minor axis of $0.045 \mathrm{~m}$ and major axis of $0.05 \mathrm{~m}$, with center at $(0.02,0)$. For the circular plate, semiuniform but irregular discretization is used, which results in a total of 18,402 material points. 

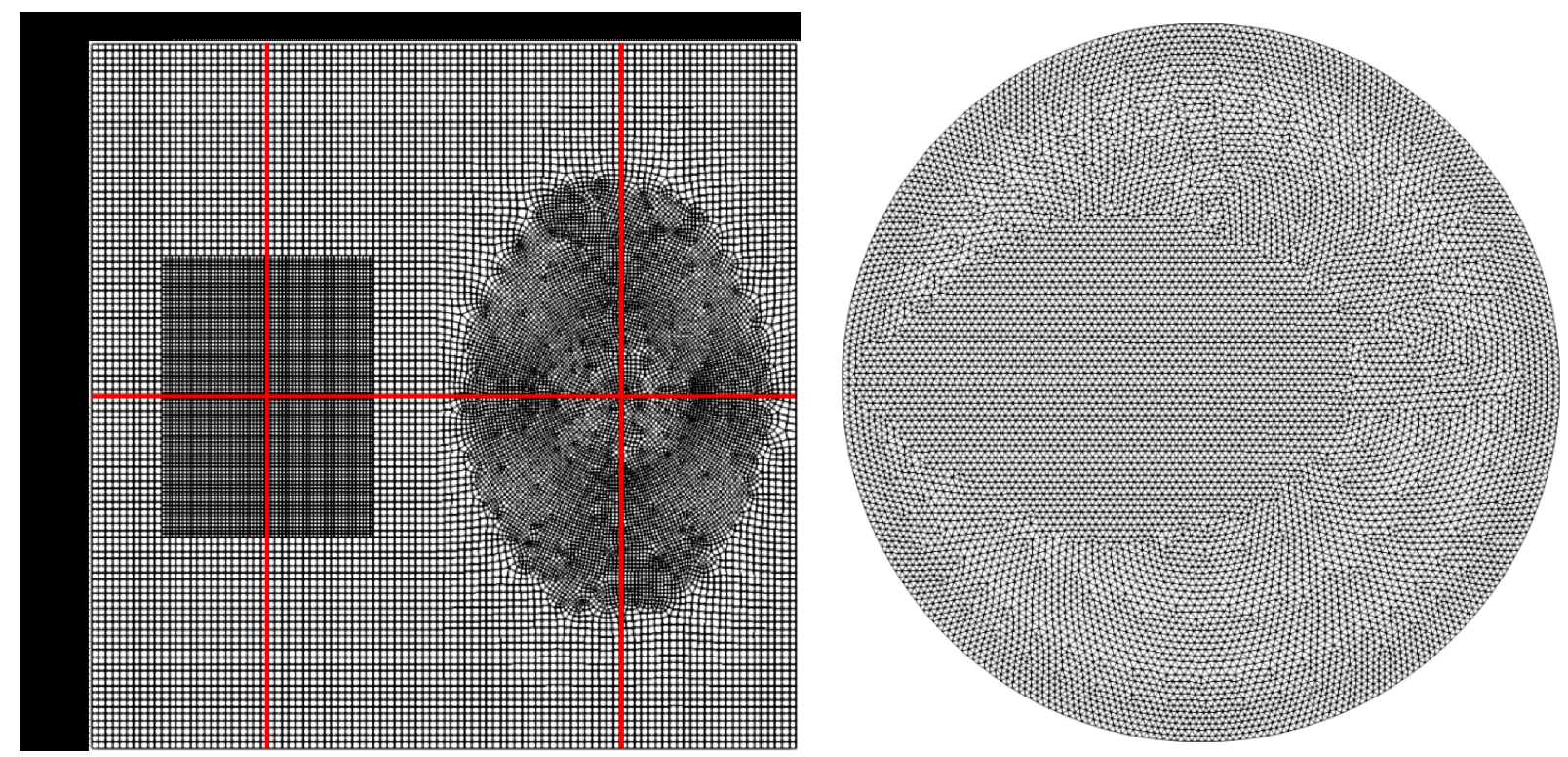

Figure 1. Spatial discretizations of geometries used in example problems: a) square plate with localized regular uniform and irregular semi-uniform discretizations, b) circular plate with irregular semi-uniform discretization

Two different configurations of horizon for each material point are considered, i.e., fixed horizon radius $\delta$ and fixed material point spacing factor $m$ or variable horizon radius. The mutual relationship completion schemes proposed in Ref. [11] is adopted for cases having variable horizon sizes. In mutual relationship completion schemes, material points are paired or unpaired by adding or deleting initially unpaired material points from their family member lists. By doing this, the unbalanced pairwise interactions between material points are completely removed. It should be noted that the addition scheme is equivalent to the dual horizon concept proposed in Ref. [17].

For discretization of square plate shown in Figure 1, when considering the material points up to the third nearest neighbor $(m=3)$, there are 296,878 bonds for addition scheme and 255,254 bonds for deletion scheme. And for the case of fixed horizon size of $0.003 \mathrm{~m}$, there are 844,972 bonds. For the circular plate, the corresponding numbers are 200,662 for addition scheme, 180,542 for deletion scheme and 300,029 for fixed horizon size of $0.172 \mathrm{~mm}$. 
The same material constants are used throughout the whole section, which are tabulated in Table 1. Abbreviations used in this section have following meanings: $\mathrm{C}$ - indicates the conventional parameters derived based on regular uniform spatial discretization are used; V- indicates the variable parameters based on general spatial discretization are used; BPD is bond-based peridynamic model [9]; OSPD is ordinary state-based peridynamic model [2]; FNOSPD is forcestabilized non-ordinary state-based peridynamic model [12]; SNOSPD is self-stabilized nonordinary state-based peridynamic model, i.e., the bond-associated peridynamic correspondence material model [13]. For SNOSPD model, the size of bond-associated horizon is the same as the corresponding material point horizon size.

Table 1. Material constants

\begin{tabular}{lc}
\hline Young's Modulus & $200 \mathrm{GPa}$ \\
\hline Poisson's Ratio & 0.345 \\
\hline Thermal Conductivity & 5.0 \\
\hline Thermal Expansion Coefficient & $1 \mathrm{E}-5$ \\
\hline Stress Free Temperature & $300 \mathrm{~K}$ \\
\hline
\end{tabular}

Case 1: Temperature/deformation distribution under applied boundary conditions

This case is developed to check the temperature and displacements distributions under applied temperature or displacement boundary conditions. The square plate is used as the solution domain in this case. Detailed verification and comparison on model prediction along three different lines are performed. The global error in the numerical prediction is quantified using a measure defined as

$$
\text { Global Error }=\frac{\int_{\Omega}\left\|u^{(a)}-u^{(p d)}\right\| d \Omega}{\int_{\Omega}\left\|u^{(a)}\right\| d \Omega}
$$


in which $\||H|$ indicates $l^{2}$-norm, the superscripts $a$ and $p d$ denote the analytical solution and peridynamic prediction respectively. $\Omega$ is the whole computational domain, and nodal integration is used.

\section{Problem 1: Heat conduction}

For this problem, temperature boundary conditions are applied, with the top edge has the value of $800 \mathrm{~K}$ and the bottom edge is $300 \mathrm{~K}$. A linearly varying temperature field is expected in the vertical direction and constant value in the horizontal direction. The predicted temperature distributions along three different lines depicted in Figure 1 for square plate using bond-based peridynamic heat conduction models are shown in Figure 2. The dotted areas indicate the local refinements in the square plate. The global errors for temperature are tabulated in Table 2.
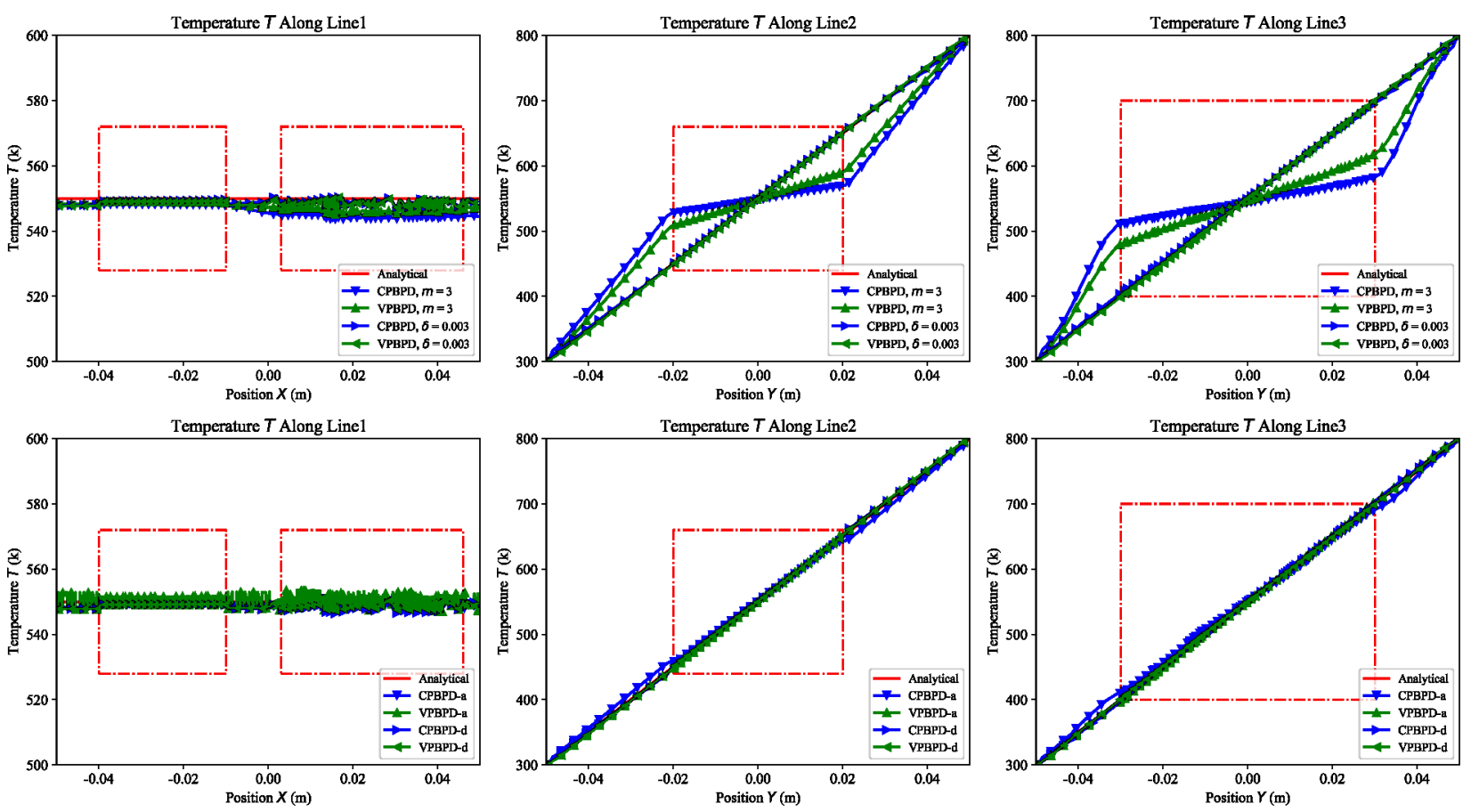

Figure 2. Distributions of temperature along Line1, Line2, Line3 using BPD

As can be seen in Figure 2, for the case of fixed material point spacing factor $m=3$, both bondbased peridynamic heat conduction models yield very poor predictions comparing to the 
analytical solution. Due to the fact that the unbalance interaction most likely exists in the transition zone between domains with different material point densities, the largest difference between prediction and analytical solution exists in these areas. For the case of fixed horizon size $\delta=0.003$, since all interactions are balanced, both models yield very accurate results. With the utilization of balance schemes to remove unbalanced interactions, the prediction accuracy improves significantly. And the deletion balance scheme outperforms the addition balance scheme. The deletion balance scheme can be used in peridynamic heat conduction models with both conventional parameter and variable parameter to yield accurate results. The global temperature errors tabulated in Table 2 for different bond-based peridynamic heat conduction models further confirms previous observations. Overall, models using variable parameter derived based on irregular non-uniform spatial discretization predict more accurate results than those using conventional parameter derived based on regular uniform spatial discretization.

Table 2. Global errors in temperature prediction for different bond-based peridynamic heat conduction models

\begin{tabular}{ccccc}
\hline Global & Constant & \multicolumn{3}{c}{ Variable Horizon } \\
\cline { 3 - 5 } Error (\%) & Horizon & NONE & ADDITION & DELETION \\
\hline CPBPD & 0.31 & 8.15 & 1.11 & 0.28 \\
\hline VPBPD & 0.21 & 4.85 & 0.31 & 0.22 \\
\hline
\end{tabular}

Problem 2: Mechanical deformation

For this problem, the top edge is applied with a displacement of $U_{y}^{t o p}=0.5 \mathrm{~mm}$ and the bottom edge is applied with displacement of $U_{y}^{\text {bottom }}=-0.5 \mathrm{~mm}$. Both centers of these two edges are fixed in the $x$ - direction. Linearly distributed displacement fields are expected in both directions. The predicted displacements along the three different lines using bond-based, state- 
based and non-ordinary state-based peridynamic mechanics models are shown in Figure 3,

Figure 4 and Figure 5, respectively. The value of $C_{Z E C}=1.0$ is used to obtained the results in Figure 5. The global errors for displacements prediction are tabulated in Table 3.
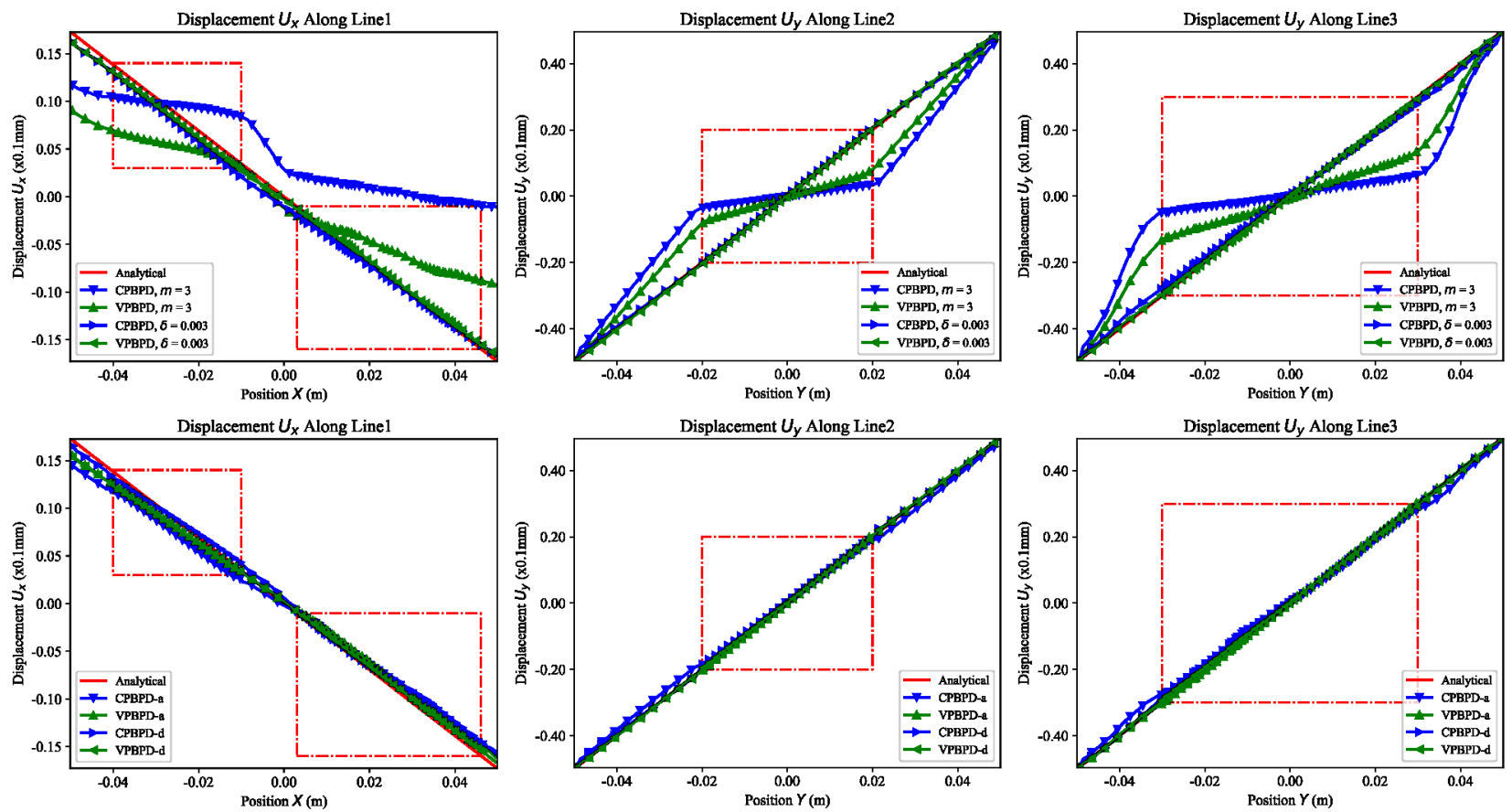

Figure 3. Distributions of displacements along Line1, Line2, Line3 using BPD
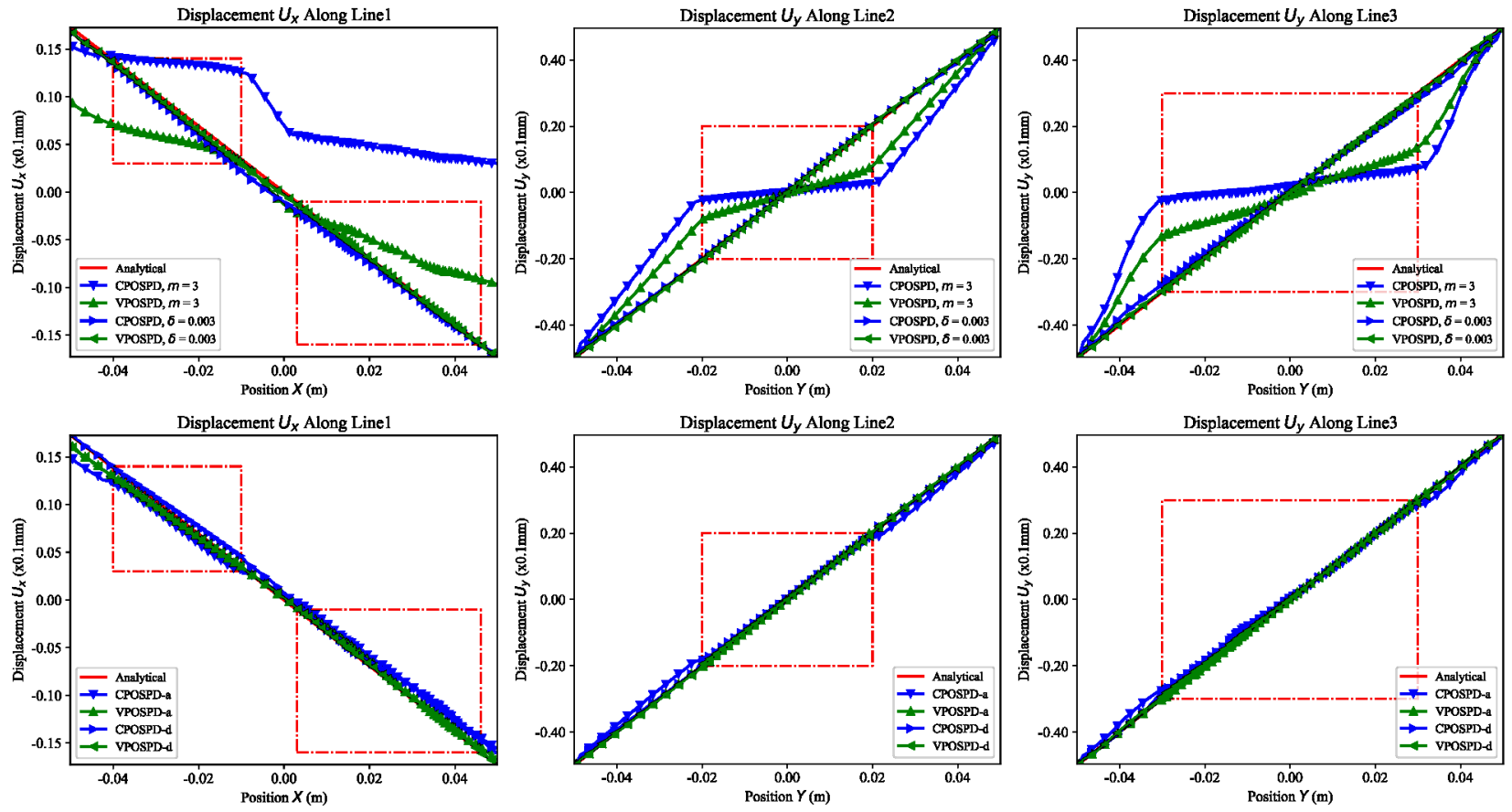

Figure 4. Distributions of displacements along Line1, Line2, Line3 using OSPD 

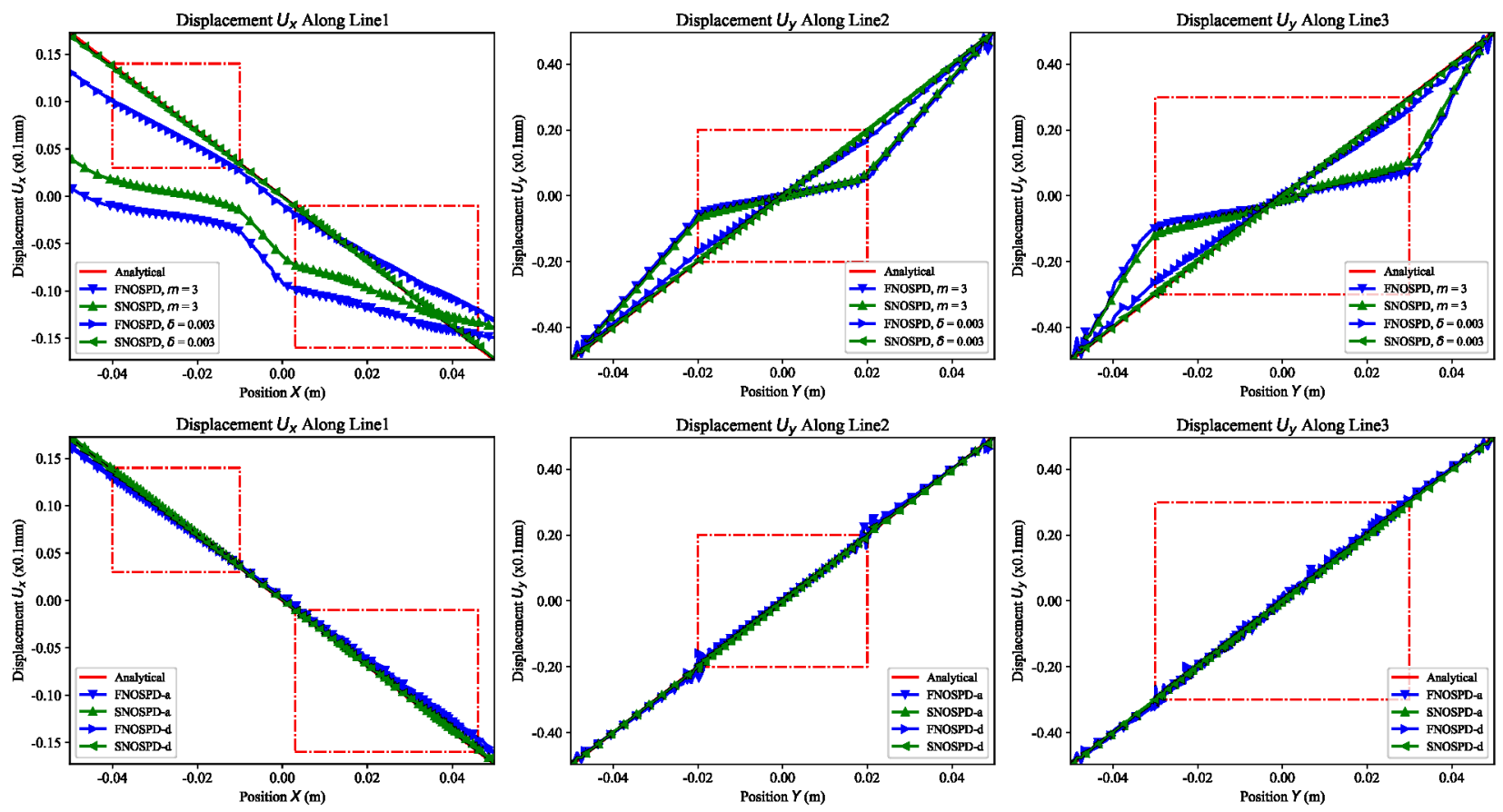

Figure 5. Distributions of displacements along Line1, Line2, Line3 using NOSPD

For all peridynamic mechanics models presented, the unbalanced interaction has more

significant effect on the lateral deformation $U_{x}$ than in peridynamic heat conduction models, as can be seen in plots along Linel in Figure 3, Figure 4, and Figure 5. The issue of limited Poisson's effect in bond-based peridynamic mechanics models also contributes to the difference between model predictions and analytical solution for the lateral deformation $U_{x}$ in Figure 3 . Similar to bond-based peridynamic mechanics models, ordinary state-based peridynamic mechanics models using variable parameters predict more accurate results than those using conventional parameters. For the conventional peridynamic correspondence material model with fictitious stabilization force, oscillations in the displacements fields still exist as can be seen from Figure 5. While for the bond-associated correspondence material model, there is no oscillation in the displacements fields and the predictions are the most accurate among all peridynamic mechanics models. 
According to the global errors in displacements predictions shown in Table 3, for models using variable parameters, the addition balance scheme slightly outperforms the deletion balance scheme. Overall, the bond-associated correspondence material model predicts the most accurate results.

Table 3. Global errors in displacements predictions for different peridynamic mechanics models

\begin{tabular}{ccccc}
\hline \multirow{2}{*}{$\begin{array}{c}\text { Global Error } \\
(\%)\end{array}$} & $\begin{array}{c}\text { Constant } \\
\text { Horizon }\end{array}$ & NONE & ADDITION & DELETION \\
\cline { 3 - 5 } CPBPD & 2.72 & 28.35 & 5.77 & 1.83 \\
\hline VPBPD & 0.96 & 19.05 & 1.09 & 1.30 \\
\hline CPOSPD & 2.76 & 25.24 & 6.53 & 1.96 \\
\hline VPOSPD & 0.98 & 18.99 & 1.12 & 1.34 \\
\hline FNOSPD & 7.1 & 22.59 & 2.31 & 2.15 \\
\hline SNOSPD & 0.81 & 20.20 & 0.56 & 0.56 \\
\hline
\end{tabular}

Case 2: Residual under globally applied temperature/displacement fields

In previous case, the model predictions under applied boundary conditions are verified with analytical solutions for irregular spatial discretization. In this case, we check the residual under prescribed linear temperature and displacement fields. It aims to check the existence of unbalanced force/heat, i.e., ghost effect, in peridynamic models when irregular spatial discretization is used in the analyses.

Problem 1: Heat conduction

The analytical temperature field $\bar{T}=5000 y+550$ from Problem 1 of Case 1 is applied to the whole solution domain of the square plate. The residual power at each material point is calculated based on the applied temperature field. Theoretically, interior material points should have zero residual power due to the heat balance at each interior material point, while material points on boundaries should have nonzero residual power. To better interpret the results from these models, the absolute value of the calculated residual power $|r|$ at each material point is 
normalized with respect to the analytical heat flux using the following expression

$$
|\bar{r}|=\frac{|r|}{\bar{q}_{y y} \cdot \sqrt{A} \cdot h}
$$

MERGEFORMAT ()

with $A$ is the area of each material point and $h$ is the plate thickness which has value of unity for two dimensional analysis.

The denominator is the applied power that would be expected at material points on the top and bottom surfaces of this model. The analytical flux $\bar{q}_{y y}=25 \mathrm{~kW} / \mathrm{m}^{2}$, so a value of 1.0 indicates an exact match to the analytical value at the top and bottom surfaces. The normalized residual power fields for different cases are shown in Figure 6 and Figure 7.
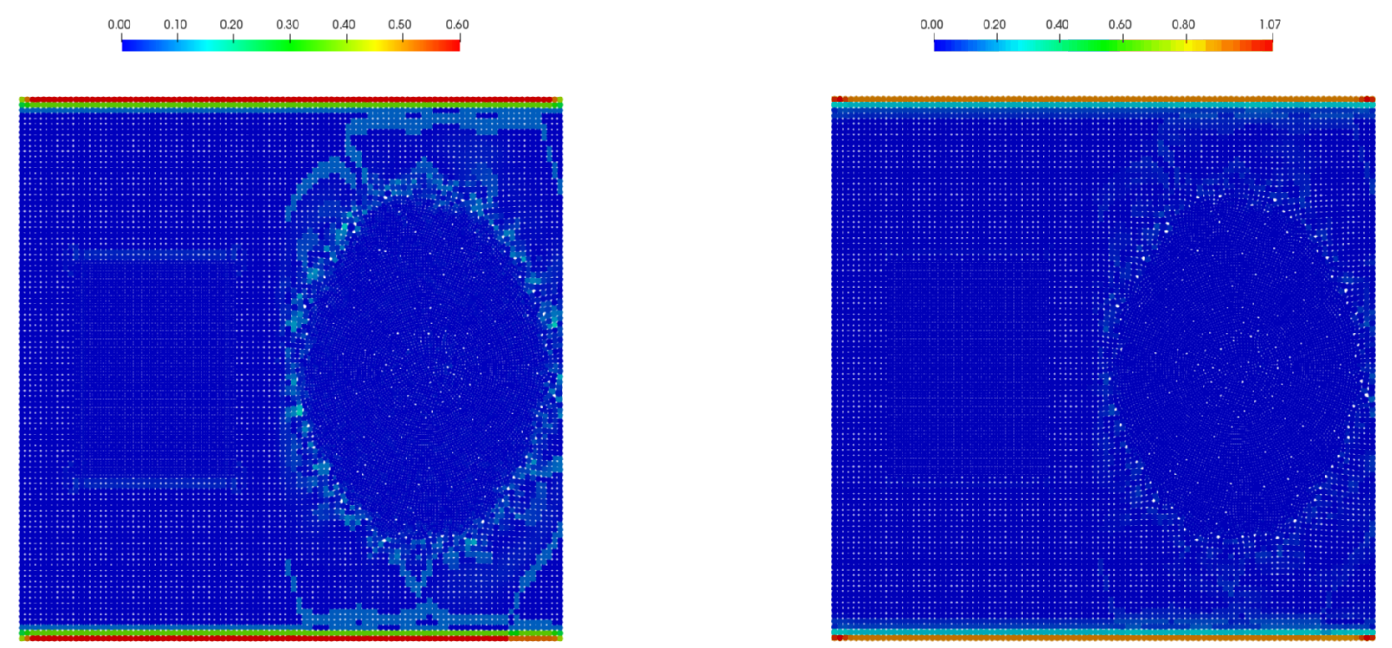

Figure 6. Normalized residual power under applied temperature field using BPD with constant horizon (left: constant parameter; right: variable parameter)

As can be seen in Figure 6, when the constant horizon size is used throughout the whole solution domain, peridynamic heat conduction model using variable parameter gives very good result and all the interior material points have zero residual power as the analytical solution. On the other hand, severe non-zero residual power exists in the peridynamic heat conduction model using constant parameter. This non-zero residual power results from the usage of constant parameter 
which is derived based on regular uniform spatial discretization. Also, for material points on the top and bottom surfaces, the residual power is far different from 1.0 for the case of constant parameter.
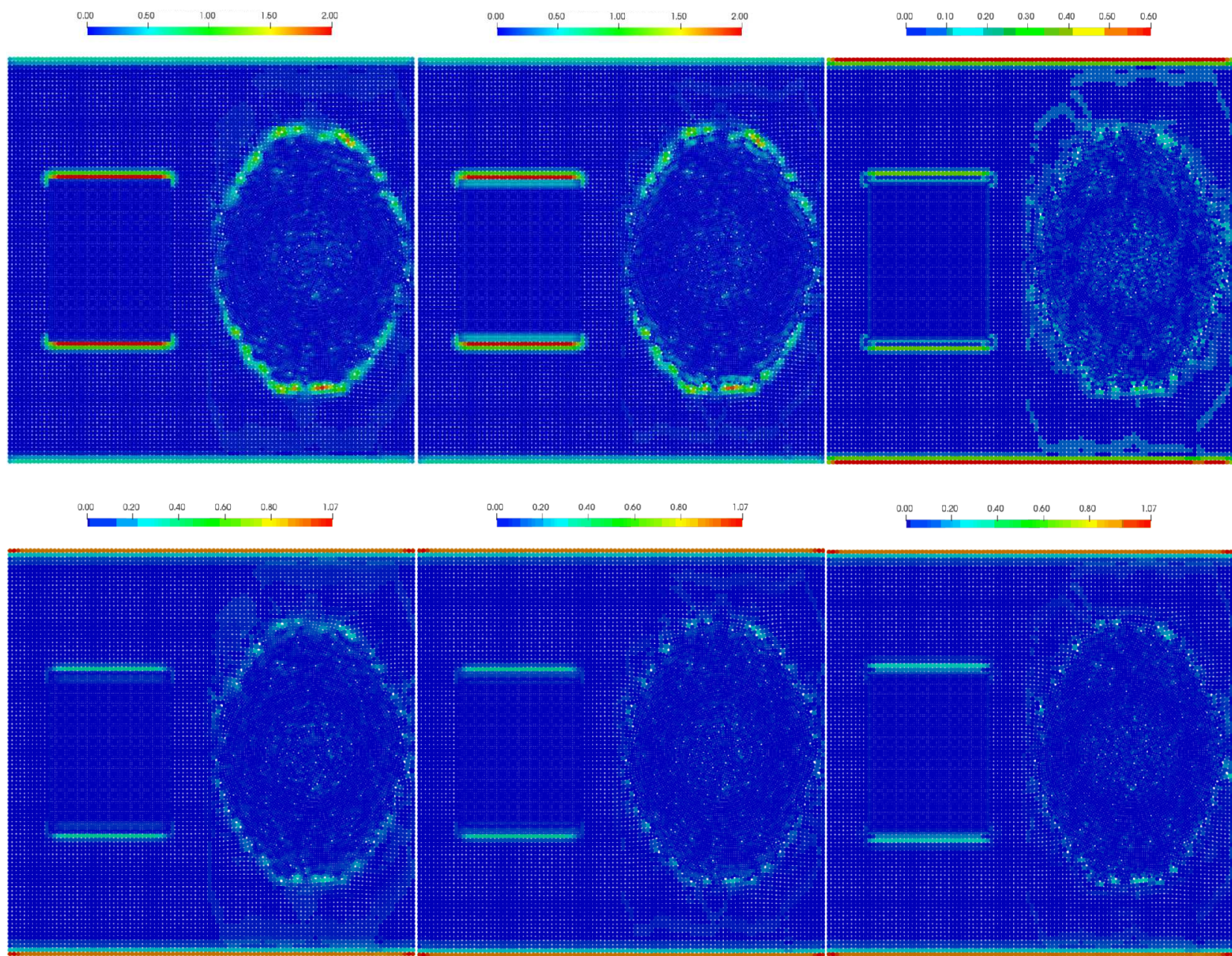

Figure 7. Normalized residual power under applied temperature field using BPD with variable horizon. (top row: constant parameter; bottom row: variable parameter; left column: no balance scheme; center column: addition balance scheme; right column: deletion balance scheme)

For cases shown in Figure 7 when variable horizon size is used, models using constant parameter give very bad results, even with balance schemes. When variable parameter is used, the results are greatly improved and no obvious difference is observed between different balance schemes. It can be concluded in this problem that for heat conduction problems the variable parameter can help to remove the unbalanced heat responses between material points. And the variable 
parameter should be used in peridynamic heat conduction model when irregular spatial discretization is used.

Problem 2: Mechanical deformation

The models employed in this case are subjected to fully prescribed linearly varying displacement fields $\bar{u}_{y}=0.001 y$ and $\bar{u}_{x}=-0.0005 x$, which correspond exactly to the analytical solutions under the loading conditions in Case 1 Problem 2. With these prescribed displacement fields, interior material points should have zero residual force due to the balance at each interior material point, while material points on boundaries should have nonzero residual forces.

The magnitude of the calculated residual force vector $|r|$ at each material point is normalized with respect to an analytical reaction force using the following expression

$$
|\bar{r}|=\frac{|r|}{\overline{S_{y y}} \cdot \sqrt{A} \cdot h}
$$

MERGEFORMAT ()

The denominator is the reaction force that would be expected at material points on the top and bottom surfaces of this model. This analytical stress solution is $\bar{S}_{y y}=65.8 \mathrm{MPa}$
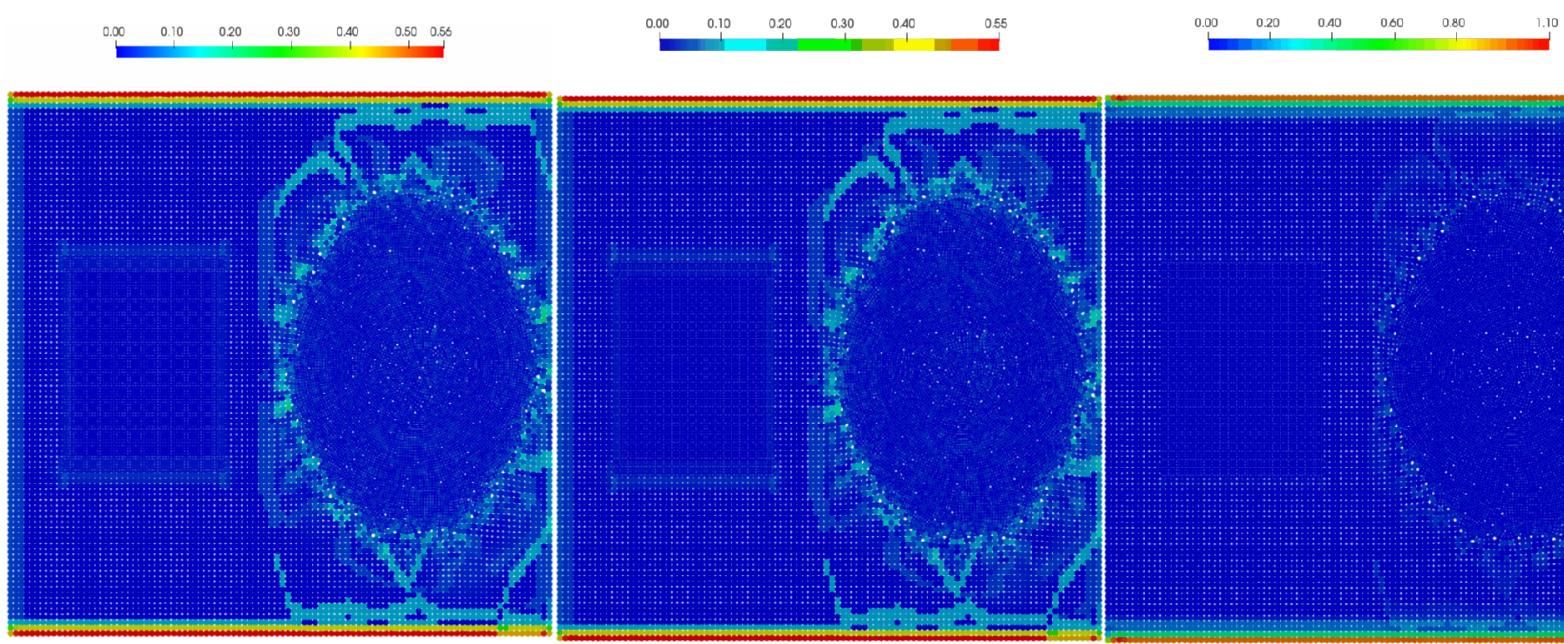


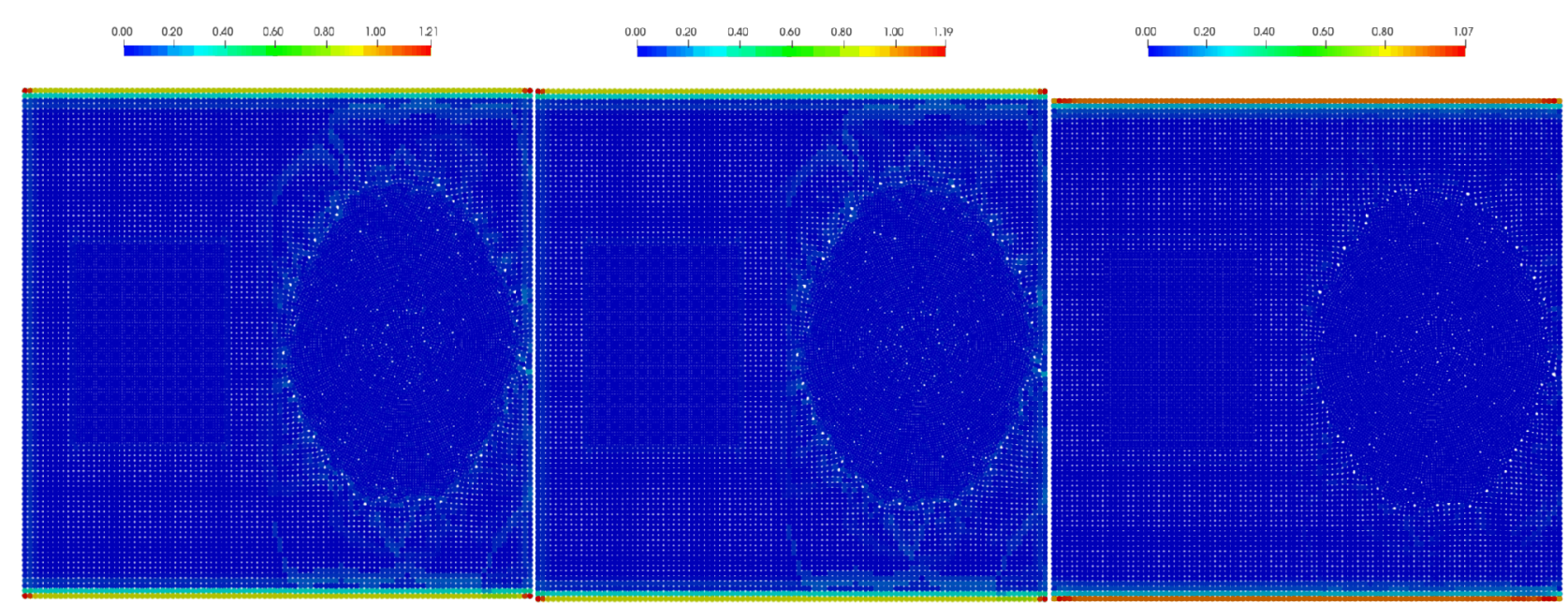

Figure 8. Normalized residual force under applied displacement field with constant horizon. (top row: constant parameter; bottom row: variable parameter; left column: BPD; center column: OSPD; right column: FNOSPD (top) and SNOSPD (bottom))

The results for different peridynamic mechanics models using constant horizon size are shown in

Figure 8. As can be seen, models using variable parameters give much better results than models with constant parameters. Among these models, the bond-associated correspondence material model yields the most accurate predictions.

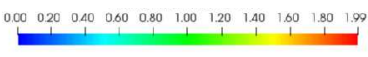

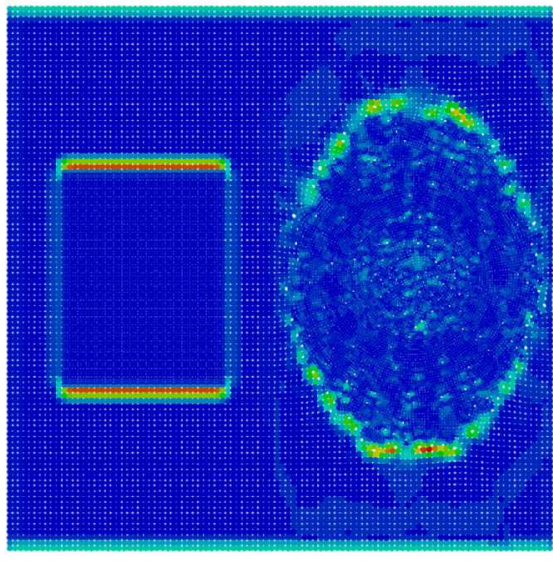

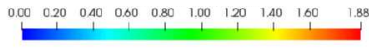

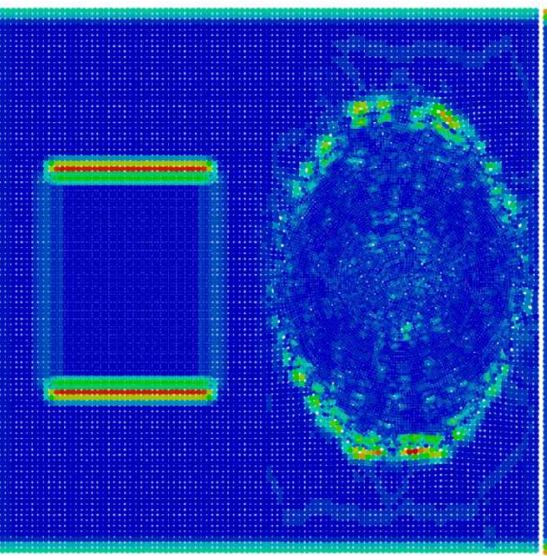

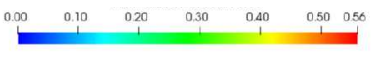

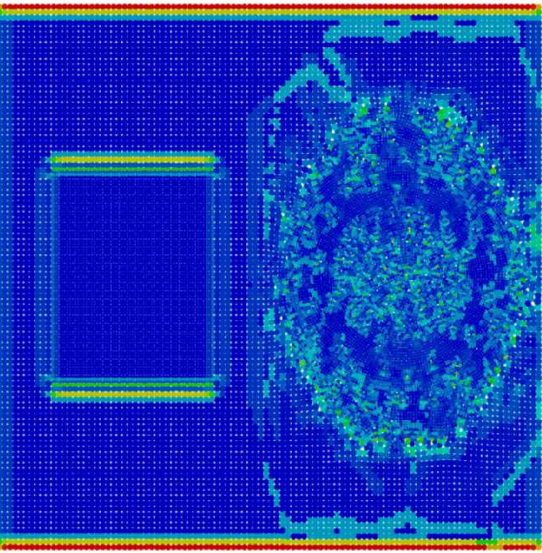



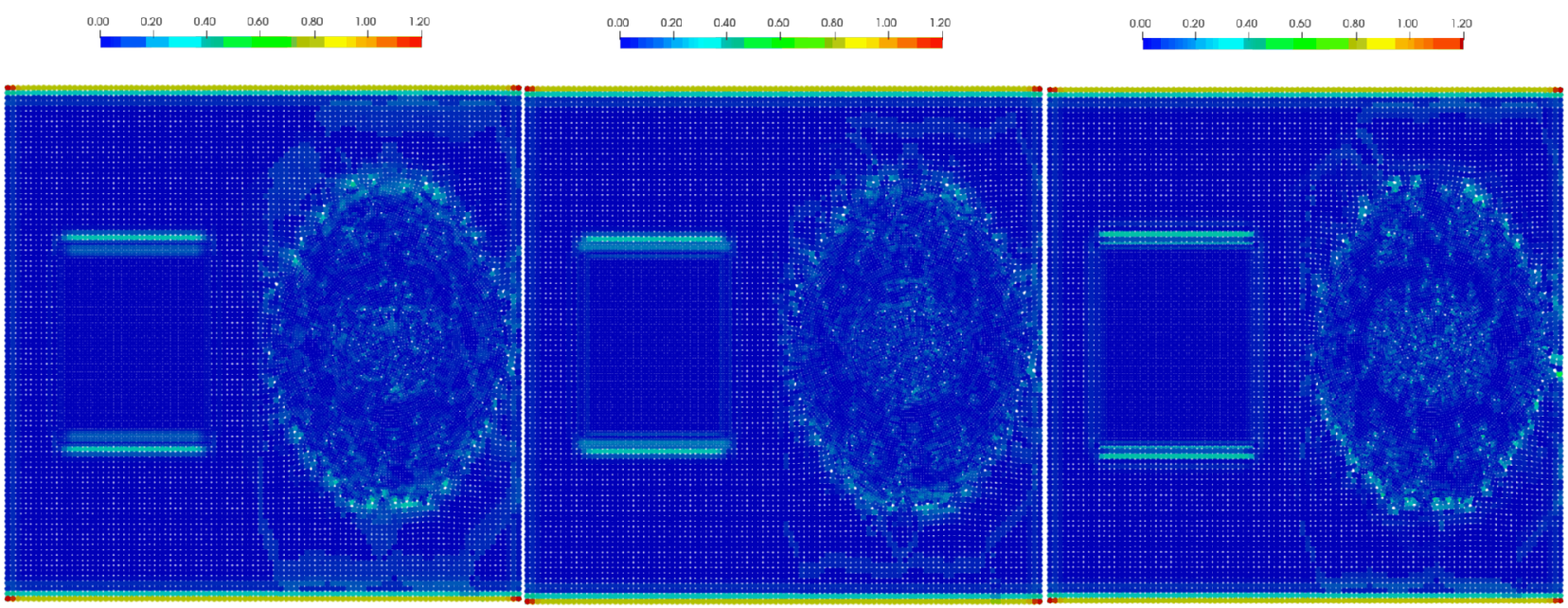

Figure 9. Normalized residual force under applied displacement field using BPD with variable horizon. (top row: constant parameter; bottom row: variable parameter; left column: no balance scheme; center column: addition balance scheme; right column: deletion balance scheme)

When variable horizon size is used, similar conclusions from constant horizon size case can be made. This can be seen from the results shown in Figure 9 and Figure 10. For bond-based and ordinary state-based peridynamic models, the non-balanced case and balanced cases using either addition or deletion scheme yield similar results in terms of residual force under globally applied displacement fields.

\begin{tabular}{cccccccc}
0.00 & 0.50 & 100 & 1.50 & 2.00 & 2.50 & 3.00 & 3.59 \\
\hline & 1 & 1 & 1 & 1 & 1 &
\end{tabular}

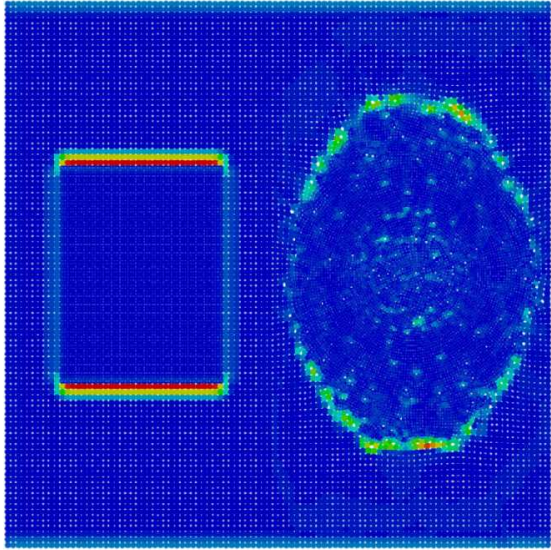

$\begin{array}{lllllllll}0.00 & 0.50 & 1.00 & 1.50 & 2.00 & 2.50 & 3.00 & 3.50 & 3.90\end{array}$

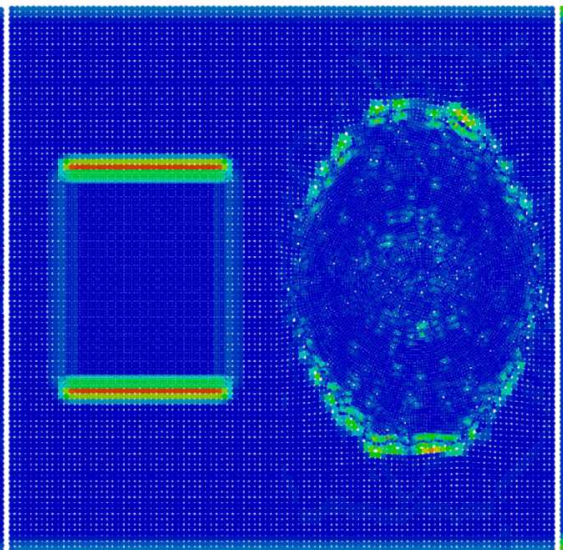

$\begin{array}{llllllll}0.00 & 0.10 & 0.20 & 0.30 & 0.40 & 0.50 & 0.60 & 0.76\end{array}$

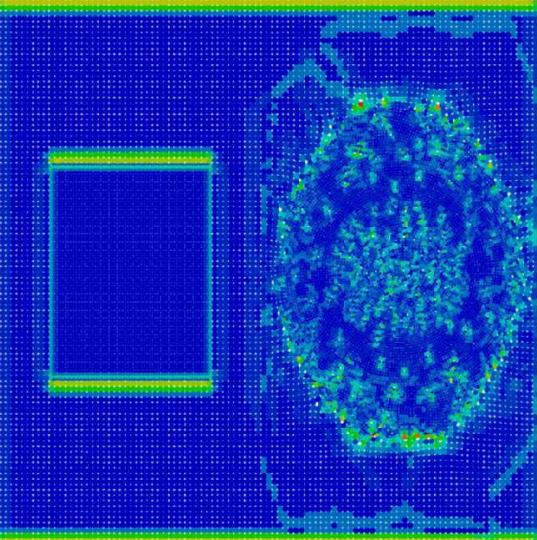



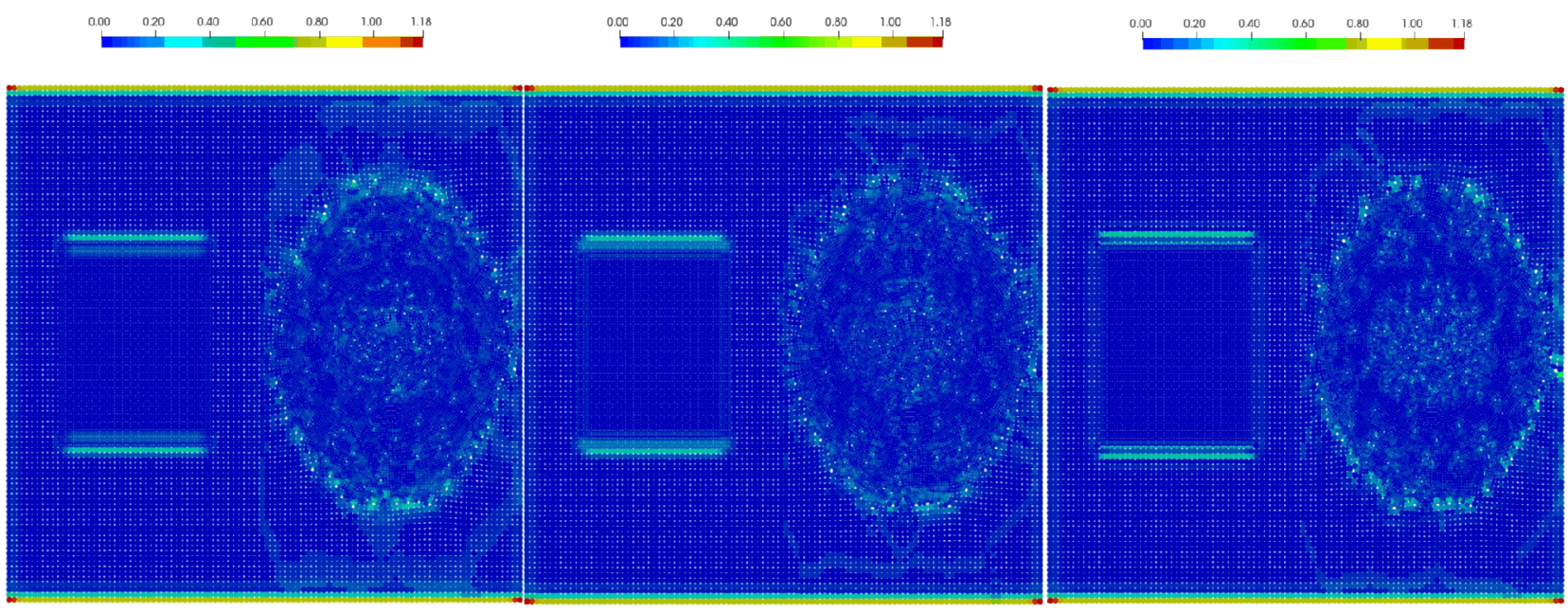

Figure 10. Normalized residual force under applied displacement field using OSPD with variable horizon. (top row: constant parameter; bottom row: variable parameter; left column: no balance scheme; center column: addition balance scheme; right column: deletion balance scheme)

For non-ordinary state-based peridynamic models, the bond-associated correspondence model gives better results than the force-stabilized model, as can be seen in Figure 11. Again, different balance schemes in non-ordinary state-based peridynamic models don't generate much difference in residual force under globally applied displacement fields.

It can be concluded again that variable parameters should be used when irregular spatial discretization is employed for bond-based and ordinary state-based peridynamic models. And the self-stabilized non-ordinary state-based model gives the most accurate prediction.
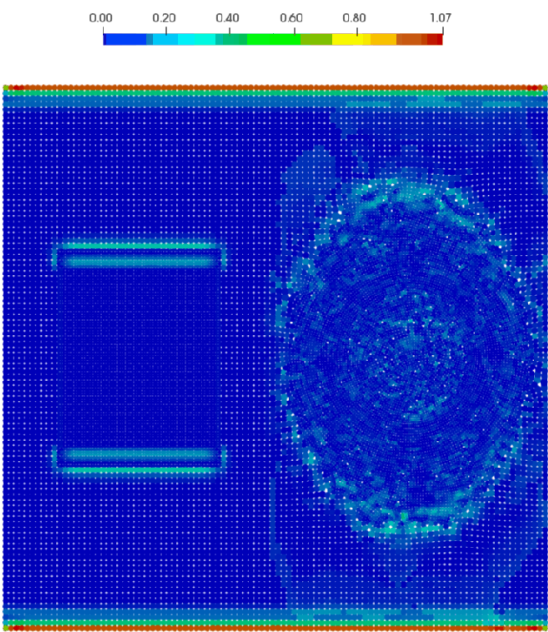
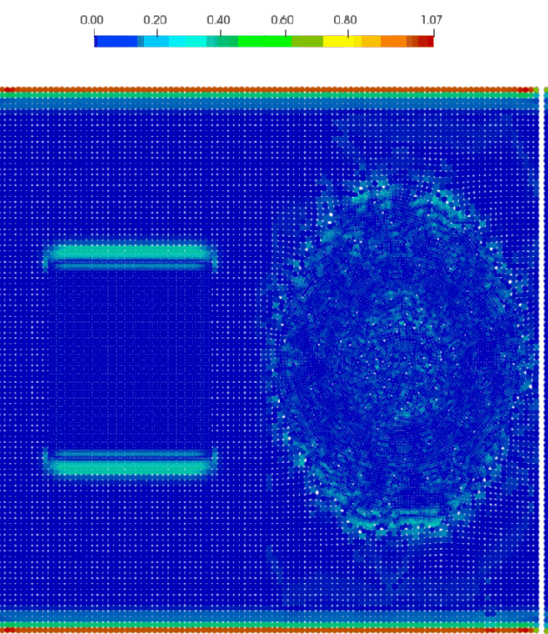
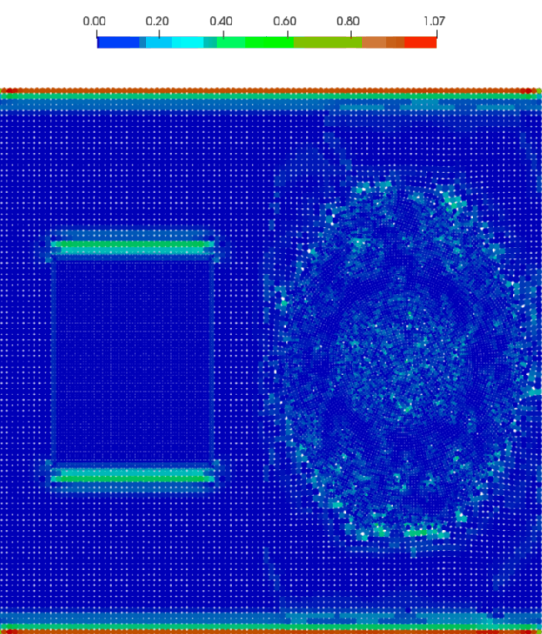

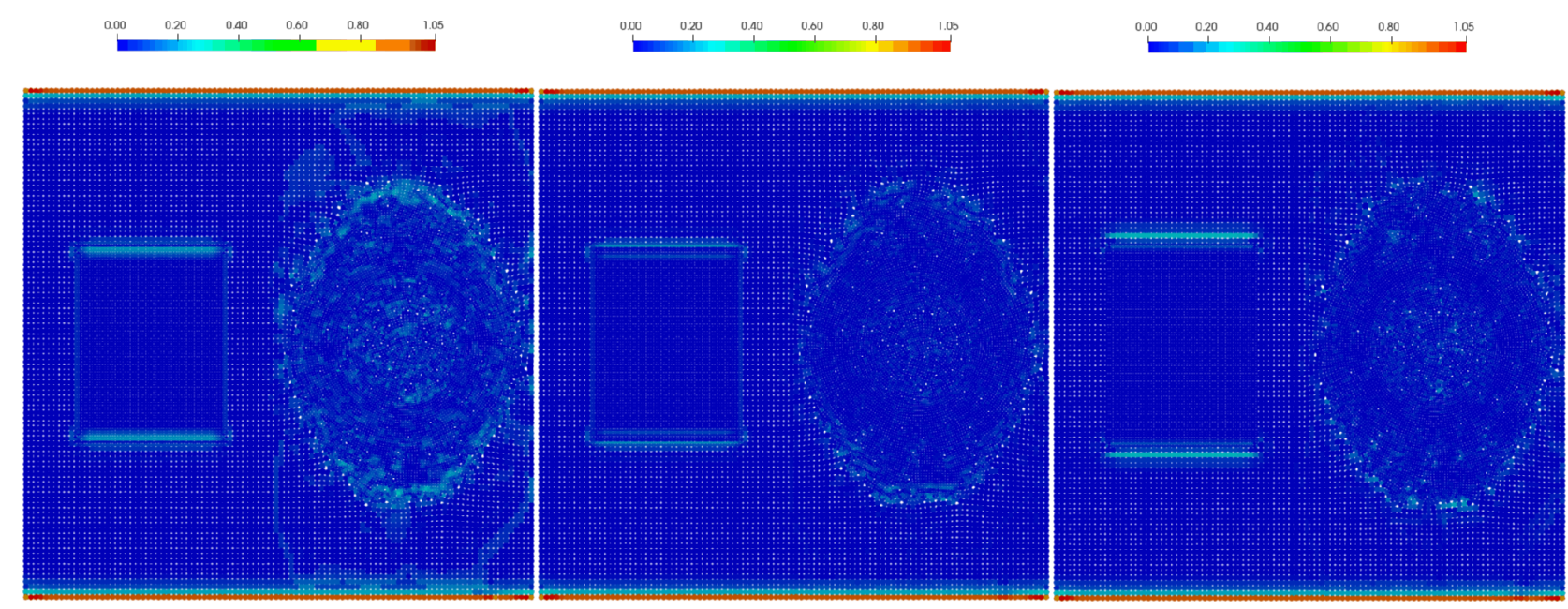

Figure 11. Normalized residual force under applied displacement field using NOSPD with variable horizon. (top row: FNOSPD; bottom row: SNOSPD; left column: no balance scheme; center column: addition balance scheme; right column: deletion balance scheme)

Case 3: Deformation under thermal loading

This case is to compare the prediction accuracy between peridynamic thermo-mechanical models for irregular spatial discretization. A circular domain discretized using irregular triangular elements as shown in Figure 1 is applied with temperature of $800 \mathrm{~K}$ at its circumference. A volumetric heat source of $473.39 \mathrm{~mW} / \mathrm{mm}^{3}$ is applied to the entire domain. The plate center is fixed and rightmost point is restrained from moving in the vertical direction. The radial temperature distributions for different bond-based models are plotted in Figure 12. As can be seen, for cases when constant horizon is used, both constant and variable parameter models yield good predictions, but variable parameter gives slightly better results. For cases when variable horizon is used, the variable parameter models outperform the constant parameter models for all cases of balance schemes. Among the two balance schemes and non-balanced case, the deletion balance scheme gives most accurate temperature prediction and the addition balance scheme yields the worst temperature prediction. This is different from the observations made in Case 1 Problem 1 for these two schemes. The author believe this difference results from the uniformity 
of the spatial discretization. For cases when non-uniform spatial discretization is used, the addition scheme gives better prediction than non-balanced case. While for cases when semiuniform spatial discretization is used, the non-balanced case gives slightly better prediction than addition scheme. But none of these two schemes give satisfactory predictions for semi-uniform spatial discretization cases.
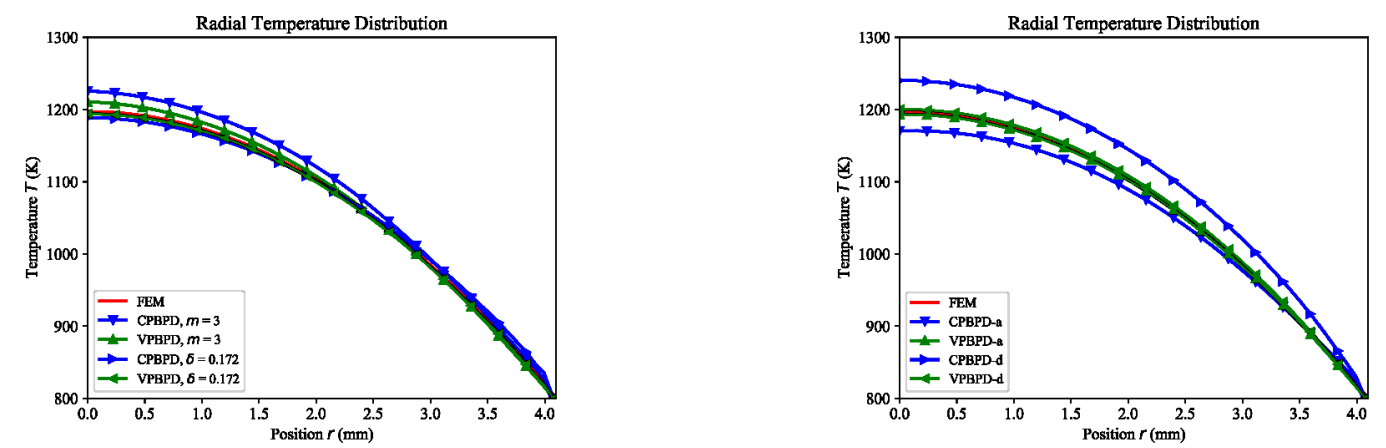

Figure 12. Distribution of temperature $T$ along radial direction using BPD

The predicted radial displacement distributions are shown in Figure 13. Similar observations for temperature field prediction apply to displacement field prediction for bond-based models, ordinary state-based models and self-stabilized non-ordinary state-based models. With exceptions to self-stabilized non-ordinary state-based models, the non-balanced case already produces very accurate results. Among these coupled thermo-mechanical models, the bond-based heat conduction model with variable parameter coupled with self-stabilized non-ordinary statebased mechanical model with deletion balance scheme yields the most accurate results.
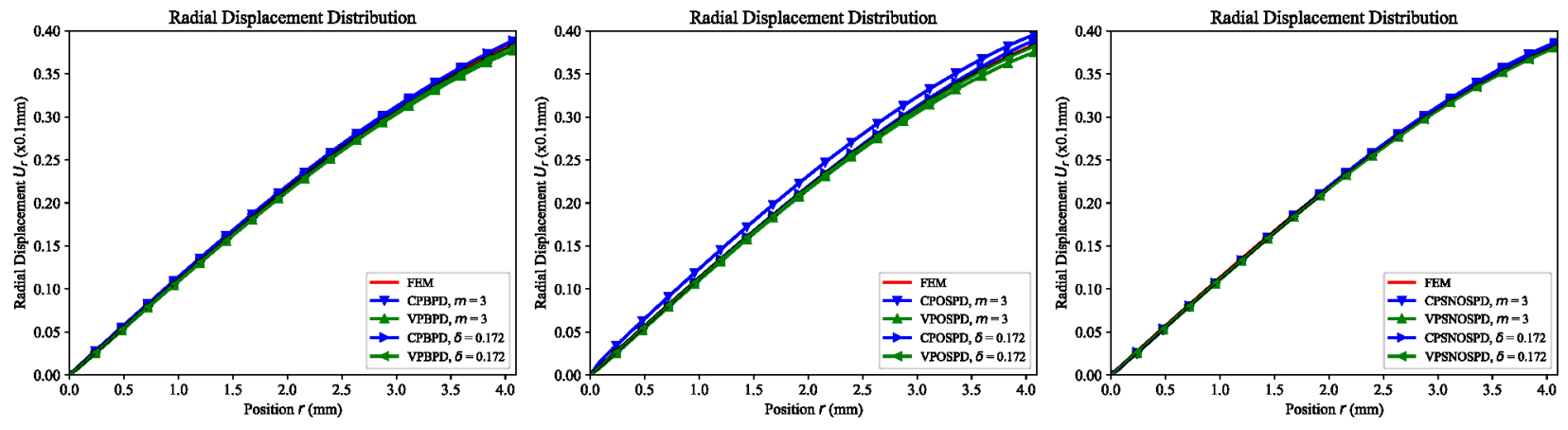

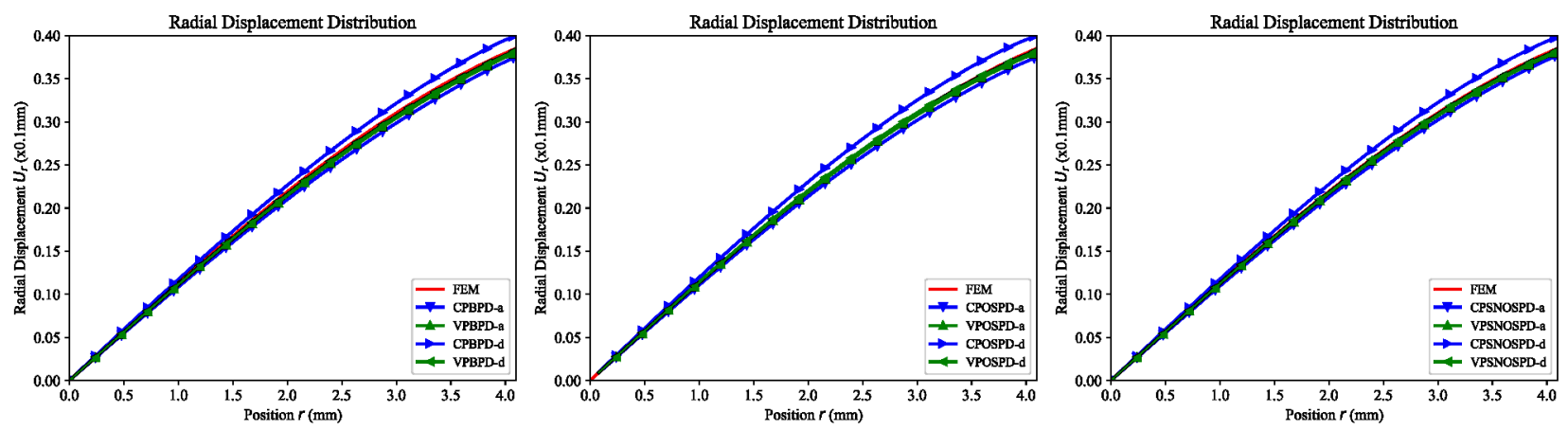

Figure 13. Distribution of displacement along radial direction using BPD, OSPD and SNOSPD

\section{Discussion and conclusion}

The reasons for applying irregular non-uniform spatial discretization in a peridynamic simulation are two-fold: first, to increase the computational accuracy in local regions of interest with minimal extra computational expense by using local mesh refinement, and second, to alleviate mesh bias effects on crack initiation and propagation. The decision on whether to use a variable horizon is motivated by these goals, and depends on the degree of uniformity of a given irregular non-uniform spatial discretization. For cases of moderate uniformity of the spatial discretization, a constant horizon should be the first choice. Otherwise, a variable horizon should be used in order to further reduce the computational expense.

In this paper, a systematic comparison study were performed to check the prediction accuracy of different bond-based, ordinary state-based and non-ordinary state-based peridynamic heat conduction and mechanics models when irregular non-uniform spatial discretization is employed. From the comparison, it is clear that the variable parameters specifically derived based on irregular non-uniform spatial discretization and interaction balancing schemes should be used when irregular spatial discretization is employed for the solution domain for bond-based and ordinary state-based peridynamic models. For non-ordinary state-based peridynamic models, i.e., correspondence material models, the interaction balancing scheme should be used for better 
prediction accuracy. For either case of spatial discretization, the bond-associated correspondence material model predicts the most accurate results. And for all peridynamic models when variable horizon size is used, the deletion scheme outperforms the addition scheme.

\section{Acknowledgement}

Work supported through the INL Laboratory Directed Research \& Development (LDRD) Program under DOE Idaho Operation Office Contract DE-AC07-05ID14517. This manuscript has been authored by Battelle Energy Alliance, LLC under Contract No. DE-AC07-05ID14517 with the U.S. Department of Energy. The United States Government retains and the publisher, by accepting the article for publication, acknowledges that the United States Government retains a nonexclusive, paid-up, irrevocable, world-wide license to publish or reproduce the published form of this manuscript, or allow others to do so, for United States Government purposes. 


\section{Reference}

1. Bobaru, F., et al., Handbook of Peridynamic Modeling. 2016, Boca Raton, FL: CRC Press.

2. Madenci, E. and E. Oterkus, Peridynamic Theory and Its Applications. 1 ed. 2014: Springer-Verlag New York. 289.

3. Silling, S.A., D.J. Littlewood, and P. Seleson, Variable horizon in a peridynamic medium. Journal of Mechanics of Materials and Structures, 2015. 10(5): p. 591-612.

4. Chen, H., et al., A Generalized 2D Non-local Lattice Spring Model for Fracture Simulation. Computational Mechanics, 2014. 54(6): p. 1541-1558.

5. Dipasquale, D., et al., Dependence of crack paths on the orientation of regular 2D peridynamic grids. Engineering Fracture Mechanics, 2016. 160: p. 248-263.

6. Henke, S.F. and S. Shanbhag, Mesh sensitivity in peridynamic simulations. Computer Physics Communications, 2014. 185(1): p. 181-193.

7. Chen, H., Y. Hu, and B.W. Spencer. A MOOSE-Based Implicit Peridynamic Thermo-Mechanical Model. in ASME 2016 International Mechanical Engineering Congress and Exposition. 2016. Phoenix, AZ: ASME.

8. Chen, H., Y. Hu, and B. Spencer. Peridynamics using Irregular Domain Discretization with MOOSEbased Implementation. in ASME 2017 International Mechanical Engineering Congress and Exposition. 2017. Tampa, FL: ASME.

9. Silling, S.A. and E. Askari, A meshfree method based on the peridynamic model of solid mechanics. Computers \& Structures, 2005. 83(17-18): p. 1526-1535.

10. Oterkus, S., E. Madenci, and A. Agwai, Peridynamic thermal diffusion. Journal of Computational Physics, 2014. 265: p. 71-96.

11. Hu, Y., et al., Thermomechanical peridynamic analysis with irregular non-uniform domain discretization. Engineering Fracture Mechanics, 2018. 197: p. 92-113.

12. Breitenfeld, M.S., et al., Non-ordinary state-based peridynamic analysis of stationary crack problems. Computer Methods in Applied Mechanics and Engineering, 2014. 272: p. 233-250.

13. Chen, H., Bond-associated deformation gradients for peridynamic correspondence model. Mechanics Research Communications, 2018. 90: p. 34-41.

14. Gaston, D., et al., MOOSE: A parallel computational framework for coupled systems of nonlinear equations. Nuclear Engineering and Design, 2009. 239(10): p. 1768-1778.

15. Van Le, Q. and F. Bobaru, Objectivity of State-Based Peridynamic Models for Elasticity. Journal of Elasticity, 2018. 131(1): p. 1-17.

16. Silling, S.A., Stability of peridynamic correspondence material models and their particle discretizations. Computer Methods in Applied Mechanics and Engineering, 2017. 322: p. 42-57.

17. Ren, H., et al., Dual-horizon peridynamics. International Journal for Numerical Methods in Engineering, 2016. 108(12): p. 1451-1476. 